\title{
Blazed-binary diffractive elements with periods much larger than the wavelength
}

\author{
Mane-Si Laure Lee, Philippe Lalanne, and Pierre Chavel \\ Laboratoire Charles Fabry de l'Institut d'Optique, Centre National de la Recherche Scientifique, BP 147, \\ F-91403 Orsay Cedex, France
}

Received November 30, 1999; accepted March 10, 2000; revised manuscript received April 5, 2000

\begin{abstract}
Blazed-binary optical elements with only binary ridges or pillars are diffractive components that mimic standard blazed-échelette diffractive elements. We report on the behavior of one-dimensional blazed-binary optical elements with local periods much larger than the wavelength. For this purpose, an approximate model based on both scalar and electromagnetic theory is proposed. The model is tested against electromagnetictheory computational results obtained for one-dimensional blazed-binary gratings with large periods. An excellent agreement is obtained, showing that the model is able to predict quantitatively the wavelength and the incidence-angle dependences of the diffraction efficiency of blazed-binary structures. (C) 2000 Optical Society of America [S0740-3232(00)01607-0]

OCIS codes: $050.1380,050.1970,050.1950$.
\end{abstract}

\section{INTRODUCTION}

Blazed-binary optical elements are diffractive components with binary profiles that achieve a high diffraction efficiency in a specified order. They are composed of subwavelength ridges, pillars, or other simple geometries carefully etched in a dielectric film. The design of blazed-binary optical elements relies strongly on the analogy between subwavelength gratings and homogeneous thin films. ${ }^{1}$ Essentially, the incident wave experiences a local effective index that depends on the local fraction of etched material. Consequently, when the size of the subwavelength features is controlled, arbitrary refractiveindex distributions can be synthesized in a single material, and "blazing" with binary features is achieved.

Recent experimental results, ${ }^{2,3}$ supported by numerical computation, ${ }^{3}$ have shown that blazed-binary optical elements composed of centrosymmetric subwavelength pillars for operation with unpolarized light offer diffraction efficiencies significantly larger than those achieved by standard blazed-échelette diffractive elements in the resonance domain. This enhanced performance has been interpreted $^{4}$ as a pillar-waveguiding effect responsible for a drastic reduction of the shadowing zone.

In this paper we are concerned with an approximate model for predicting the wavelength and incidence-angle dependences of the performance of blazed-binary structures with local periods much larger than the wavelength, i.e., with a coded phase that is slowly varying at the scale of the wavelength. This situation is of great practical interest. For instance, slowly varying phase functions are encountered for the central zones of diffractive lenses or for geometrical aberration compensation and achromatization of optical systems. The approximate model is described in Section 2. The analysis is restricted to onedimensional (1-D) blazed-binary structures composed of ridges operating for TM (magnetic vector parallel to the ridges) or TE (electric vector parallel to the ridges) polarizations. In Section 3 the approximate model is tested against electromagnetic theory obtained for 1-D blazedbinary gratings with periods up to 50 wavelengths. It is shown that the approximate model is able to predict accurately the wavelength and incidence-angle dependences of the diffraction efficiency.

\section{APPROXIMATE MODEL}

Before describing the approximate model, we first briefly outline the main steps leading to the design of blazedbinary diffractive elements. Once an unwrapped phase function $\phi(x)$ is specified at a given (nominal) wavelength $\lambda_{0}$ and at a given angle of incidence (in general, zero) [see Fig. 1(a)], the corresponding diffractive phase function $\Psi(x)$, equal to $\phi(x)$ modulo $2 \pi$, is generated [see Fig. 1(b)]. This diffractive phase function is then sampled on a regular grid. The sampling period, or, equivalently, the distance between two adjacent subwavelength ridges, is denoted by $\Lambda_{s}$ and is shown in the enlarged window of Fig. 1(c). A calibration curve ${ }^{3}$ that relates the phase delay for a given etched depth, or, equivalently, the effective index, to the fraction of material removed is then used to associate a specific microstructure fill factor with a specific phase shift $\left[\Psi_{1}\right.$ in Fig. 1(c)]. The key point for the following is that a systematic relationship exists between the designed blazed-binary structures and the unwrapped phase function. As illustrated in Figs. 1(b) and 1(c), whenever the unwrapped phase shift is equal, modulo $2 \pi$, to an arbitrary value $\Psi_{1}$, a specific subwavelength ridge with a specific fill factor is generated locally. The model we propose strongly exploits this relationship. It relies on both scalar and electromagnetic theory.

\section{A. Scalar Wave Approximation}

Because of the slowly varying assumption, we first define a complex-amplitude transmittance for the blazed-binary diffractive element. As is usually done for calculating propagation in Fourier optics, this transmittance is de- 
(a)

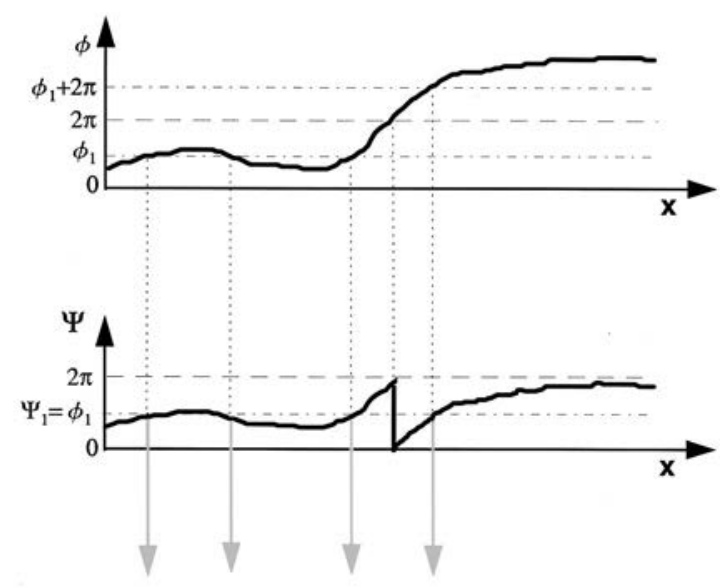

(c)

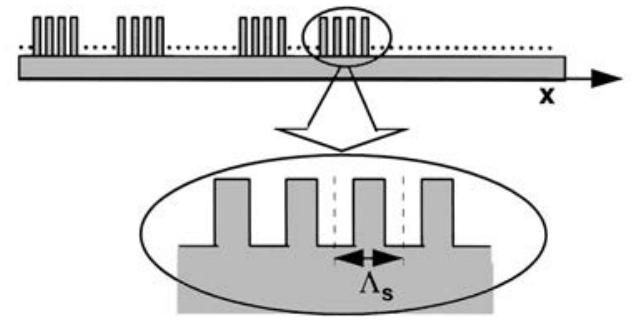

Fig. 1. Encoding a phase transfer function with blazed-binary structures: (a) unwrapped phase transfer function, (b) phase transfer function of the corresponding diffractive optical element, and (c) corresponding blazed-binary structure.

fined as the ratio between the transmitted $U_{t}$ and the incident $U_{i}$ complex scalar wave functions. These two functions are such as the square of their modulus represent intensities. Because of the systematic relationship we mentioned earlier, the transmittance $t(\phi)$ of the blazed-binary diffractive element is a $2 \pi$-periodic function of the unwrapped phase $\phi$. Thus it can be expanded in a Fourier series:

$$
t(\phi)=\sum_{-\infty}^{+\infty} c_{n} \exp (j n \phi)
$$

The $c_{n}$ coefficients are given by

$$
c_{n}=\frac{1}{2 \pi} \int_{0}^{2 \pi} t(\phi) \exp (-j n \phi) \mathrm{d} \phi,
$$

and the $n$ th-transmitted-order diffraction efficiency $\eta_{n}$ (we are generally concerned with $\eta_{1}$ ) is given by

$$
\eta_{n}=\left|c_{n}\right|^{2} .
$$

The expansion in Eq. (1) describes the transmittance of the blazed-binary structures as a coherent superposition of scalar wave functions with a weighted intensity distribution given by the $\eta_{n}$ 's. The diffraction efficiencies $\eta_{n}$ are computed through the numerical integration of the right-hand side of Eq. (2). This integration requires the knowledge of the complex-amplitude transmittance $t(\phi)$; this will be explained in Subsection 2.B.

\section{B. Zero-Order Local Grating Approximation}

We assume that the blazed-binary structure is illuminated by an incident plane wave $A_{i}$ in a medium of refrac- tive index $n_{1}$. If we denote by $\theta_{1}$ the angle of incidence, this plane wave can be written as

$$
A_{i}=\exp \left[-j k_{0} n_{1}\left(\sin \theta_{1} x+\cos \theta_{1} z\right)\right],
$$

where $A_{i}$ denotes the $y$ component of the magnetic field for TM polarization or the $y$ component of the electric field for TE polarization. In Eq. (4) $k(k=2 \pi / \lambda)$ denotes the modulus of the incident wave vector in the vacuum. Note that the wavelength $\lambda$ of the incident plane wave may be different from the nominal wavelength $\lambda_{0}$. Locally, because the blazed-binary diffractive elements profile is slowly varying, the incident plane wave $A_{i}$ sees a zeroorder lamellar grating at the scale of several wavelengths. By a zero-order grating, we mean a grating whose orders are all evanescent except the zero-reflected and zero-transmitted ones. The response of this zeroorder grating is easily computed with electromagnetic grating theory. The only contribution in the transmitted far field is the zero-order homogeneous plane wave $A_{t}$ given by

$$
A_{t}=T_{0}(\phi) \exp \left[-j k_{0}\left(n_{1} \sin \theta_{1} x+n_{3} \cos \theta_{3} z\right)\right] .
$$

In Eq. (5) $n_{3}$ is the refractive-index of the emerging medium and $\theta_{3}$ is given by the Snell law, $n_{1} \sin \theta_{1}$ $=n_{3} \sin \theta_{3}$. In Eq. (5) $T_{0}(\phi)$ is the normalized amplitude of the zero-transmitted order.

We now derive the expression for the complexamplitude transmittance $t(\phi)$. The electromagnetic quantity related to the light intensity $U_{t} U_{t}^{*}$ or $U_{i} U_{i}^{*}$ is the $z$ component $S_{z}$ of the time-averaged Poynting vector. ${ }^{5}$ For TE waves, $S_{z}$ is proportional to $n_{1} \cos \theta_{1}$ for the incident wave and $n_{3} \cos \theta_{3} T_{0}(\phi) T_{0}^{*}(\phi)$ for the zeroorder transmitted wave (the omitted proportional factor is simply $\left.1 / 2 c \mu_{0}\right)$. Thus we can define $U_{i}$ and $U_{t}$ by $U_{i}$ $=\left(n_{1} \cos \theta_{1}\right)^{1 / 2} A_{i}$ and $U_{t}=\left(n_{3} \cos \theta_{3}\right)^{1 / 2} A_{t}$, and the complex-amplitude transmittance for TE waves is

$$
t(\phi)=\left(\frac{n_{3} \cos \theta_{3}}{n_{1} \cos \theta_{1}}\right)^{1 / 2} T_{0}(\phi) .
$$

Similarly, for TM waves, $S_{z}$ is proportional to $\cos \theta_{1} / n_{1}$ and $\left(\cos \theta_{3} / n_{3}\right) T_{0}(\phi) T_{0}^{*}(\phi)$ for the incident and the zeroorder transmitted waves, respectively. Thus $U_{i}$ $=\left(\cos \theta_{1} / n_{1}\right)^{1 / 2} A_{i}, \quad U_{t}=\left(\cos \theta_{3} / n_{3}\right)^{1 / 2} A_{t}, \quad$ and the complex-amplitude transmittance is

$$
t(\phi)=\left(\frac{n_{1} \cos \theta_{3}}{n_{3} \cos \theta_{1}}\right)^{1 / 2} T_{0}(\phi) .
$$

As shown by Eqs. (6) and (7), the numerical integration of Eq. (2) requires the computation of the normalized amplitude $T_{0}(\phi)$ of the zero-transmitted order of many zeroorder gratings. The analysis of the two-dimensional (2-D) blazed-binary structures requires electromagnetic computations (the $T_{0}$ 's) performed only on 1-D zero-order gratings. Similarly, the analysis of three-dimensional (3-D) blazed-binary structures (not investigated in this paper), like those considered in Ref. 3, would require electromagnetic computations performed only on 2-D zeroorder gratings. Although we restricted the analysis to the transmitted far field for the sake of conciseness, note 
that the reflected field is straightforwardly obtained through the computation of the normalized amplitudes of the zero-reflected order.

\section{TESTING THE APPROXIMATE MODEL}

In this section we test the approximate model against electromagnetic theory. In principle, the approximate model is valid for any blazed-binary structure encoding an unwrapped phase function that is slowly varying at the scale of the wavelength. However, because full electromagnetic analysis of arbitrary blazed-binary structures (like lenses or spot array generators) with finite apertures is unpractical with today's computers, the model is tested against electromagnetic results obtained only for blazed-binary gratings with periods up to 50 wavelengths. These blazed-binary gratings are assumed to be composed of grooves etched into a $\mathrm{TiO}_{2}$ layer (the refractive index is equal to 2.3$)$ coated on a glass substrate $\left(n_{1}=1.52\right)$. They are illuminated from the glass substrate, and they are designed for a normally incident plane wave at the nominal wavelength of $\lambda_{0}=633 \mathrm{~nm}$. We partly take into account fabrication constraints by limiting the maximum encoded effective index to a value of $n_{\max }=1.67$. This value is the one used in Ref. 3 for blazed-binary structures composed of subwavelength pillars. The depth of the blazed-binary gratings is $\lambda_{0} /\left(n_{\max }-1\right)$. Fabrication constraints for small fill factors (small effective indices) are not considered in this theoretical study, but they could have been easily incorporated by clipping the function $T_{0}(\phi)$ for small $\phi$ values. The rigorous coupled-wave analysis ${ }^{6}$ (RCWA) and its enhanced version $^{7}$ for TM polarization are used for the computations.

\section{A. Normal Incidence and Nominal Wavelength}

In this subsection we assume that the blazed-binary gratings are illuminated at normal incidence from the glass substrate and at the nominal wavelength $\lambda_{0}$ of the design. Let us consider first the TM polarization case. Figure 2 shows three examples of the function $T_{0}(\phi)$ for three different sampling periods $\Lambda_{s}$. The solid, dashed, and dotted curves correspond to $\Lambda_{s}=\lambda_{0} / 2, \lambda_{0} / 5$, and $\lambda_{0} / 10$, respectively. We note only a small difference among these three curves. This small difference reflects in the corresponding values for $\eta_{1}$ in Eq. (3). that are nearly identical: $\eta_{1}=97.1,97.1$, and $96.9 \%$ for $\Lambda_{s}=\lambda_{0} / 2, \lambda_{0} / 5$, and $\lambda_{0} / 10$, respectively. These three values are represented as horizontal lines in Fig. 3. They correspond to the firsttransmitted-order diffraction efficiency of an arbitrary blazed-binary diffractive element (lenses, kinoforms) with an infinitely slowly varying phase function. Thus they also correspond to the first-transmitted-order diffraction efficiencies of blazed-binary gratings with infinitely large periods. In addition, the first-transmitted-order diffraction efficiency of blazed-binary gratings computed with the RCWA are plotted as a function of the period-towavelength ratio. Squares, triangles, and stars are obtained for $\Lambda_{s}=\lambda_{0} / 2, \lambda_{0} / 5$, and $\lambda_{0} / 10$, respectively. In Fig. 3 it is noticeable that although the sampling period has a weak effect on the asymptotic efficiencies, it has a strong effect on the performance of the blazed-binary

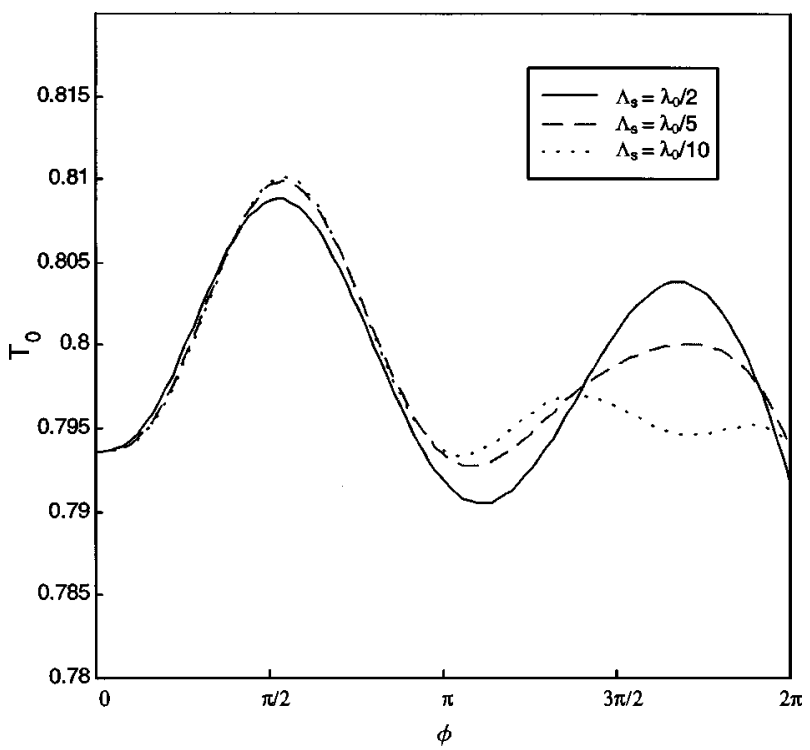

Fig. 2. Modulus of the normalized amplitude of the zerotransmitted order for three sampling periods $\left[\Lambda_{s}=\lambda_{0} / 2\right.$ (solid curve), $\Lambda_{s}=\lambda_{0} / 5$ (dashed curve), and $\Lambda_{s}=\lambda_{0} / 10$ (dotted curve)] and for TM polarization. Because normal incidence from the glass substrate at the nominal wavelength is assumed for the computation, the argument of $T_{0}(\phi)$ is simply $\phi$ in this specific case.

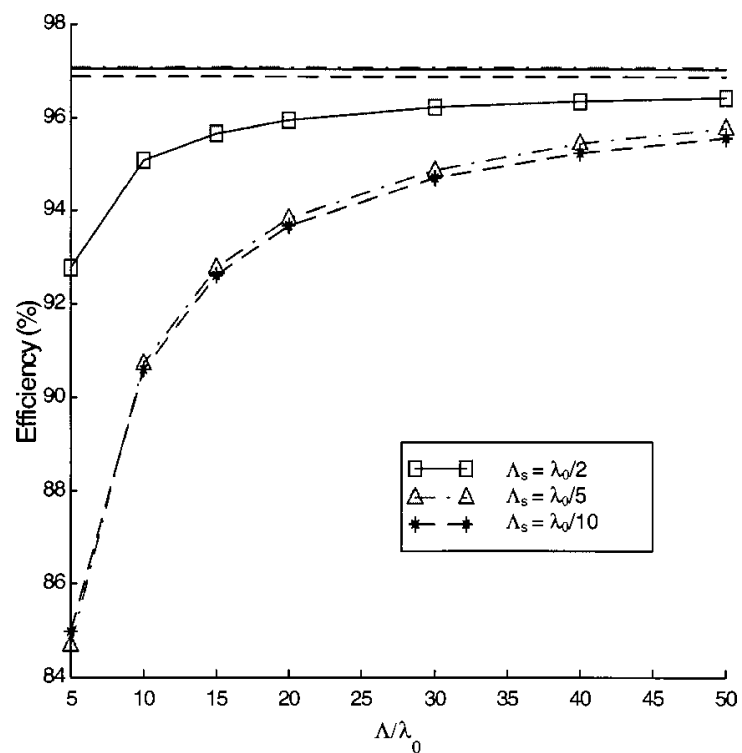

Fig. 3. One-dimensional blazed-binary gratings for TM polarization. First-transmitted-order efficiency as a function of the period-to-wavelength ratio for a normal incidence from the glass substrate. The horizontal lines correspond to the asymptotic predictions for $\Lambda / \lambda_{0} \rightarrow \infty$.

gratings for moderately large period-to-wavelength ratios. The best performance is achieved for the largest sampling period. The reason comes from a waveguiding effect, as explained in Ref. 4.

The overall shapes for the curves in Fig. 3 indicates that the predicted asymptotic values for $\eta_{1}$ are qualitatively correct. To test the model more quantitatively, we now compare the asymptotic predictions with the extrapolated values obtained from the RCWA numerical results. 
For this purpose, we use a polynomial interpolation based on the assumption that the efficiency is a polynomial function of the wavelength-to-period ratio,

$$
\eta_{1}\left(\frac{\lambda_{0}}{\Lambda}\right)=\sum_{i=0}^{N-1} a_{i}\left(\frac{\lambda_{0}}{\Lambda}\right)^{i},
$$

where $N$ is a natural number related to the number of known (calculated by the electromagnetic theory) values. The efficiency for infinitely large periods is simply given by $a_{0}$. To compute the $a_{i}$ coefficients, we use Neville's algorithm. ${ }^{8}$ This algorithm is a recursive method that has the advantage of allowing us to calculate an error estimation of the extrapolated values. For the extrapola-

Table 1. TE Polarization. Comparison of the Asymptotic Values $\eta_{1}$ of the First-TransmittedOrder Efficiency Predicted by the Approximate Model and the Extrapolated Values $a_{0}$ Obtained from the RCWA Results of Fig. $3^{a}$

\begin{tabular}{lccc}
\hline Value & $\begin{array}{c}\Lambda_{s}=\lambda_{0} / 2 \\
(\%)\end{array}$ & $\begin{array}{c}\Lambda_{s}=\lambda_{0} / 5 \\
(\%)\end{array}$ & $\begin{array}{c}\Lambda_{s}=\lambda_{0} / 10 \\
(\%)\end{array}$ \\
\hline$\eta_{1}$ & 97.1 & 97.1 & 96.9 \\
$a_{0}$ & 97.2 & 96.7 & 97.0 \\
(error estimate) & $(0.6)$ & $(0.6)$ & $(0.2)$ \\
\hline
\end{tabular}

${ }^{a}$ The values given in parenthesis correspond to the estimated errors of the coefficients $a_{0}$.

Table 2. Same As Table 1 Except for TE Polarization and RCWA Values Obtained from Fig. 4

\begin{tabular}{lccc}
\hline Value & $\Lambda_{s}=\lambda_{0} / 2$ & $\Lambda_{s}=\lambda_{0} / 5$ & $\Lambda_{s}=\lambda_{0} / 10$ \\
$(\%)$ & $(\%)$ & 96.7 \\
\hline$\eta_{1}$ & 96.2 & 96.7 & 96.6 \\
$a_{0}$ & 96.3 & 96.9 & $(0.1)$ \\
(error estimate) & $(0.1)$ & $(0.3)$ & \\
\hline
\end{tabular}

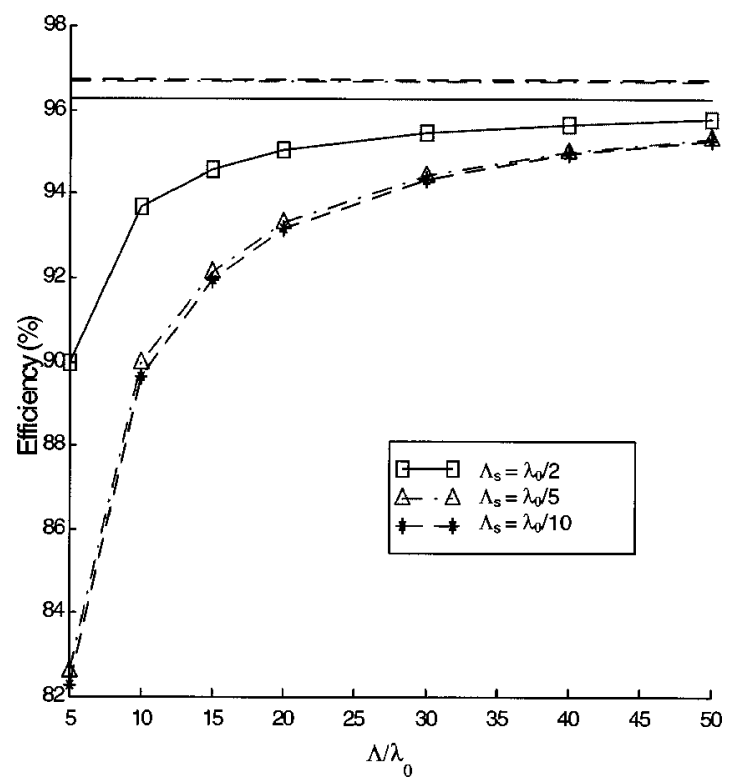

Fig. 4. Same as Fig. 3 but for TE polarization.

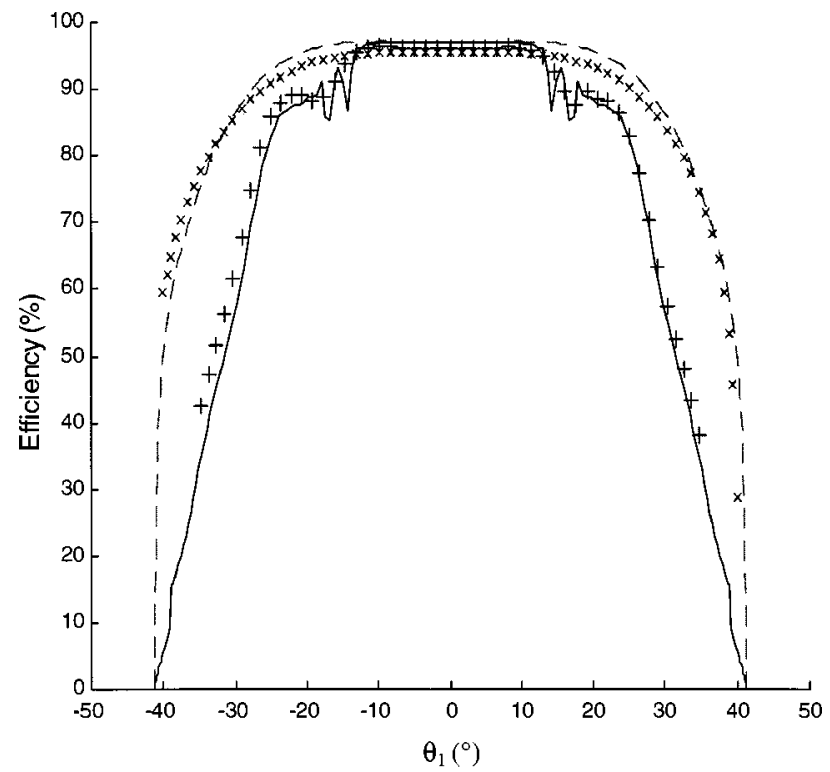

Fig. 5. First-transmitted-order diffraction efficiency versus angle of incidence $\theta_{1}$ in glass for TM polarization. Solid $\left(\Lambda_{s}\right.$ $\left.=\lambda_{0} / 2\right)$ and dashed $\left(\Lambda_{s}=\lambda_{0} / 5\right)$ curves, asymptotic predictions of the model for $\Lambda / \lambda_{0} \rightarrow \infty$. Pluses and crosses, RCWA computational results obtained for $50 \lambda_{0}$-period blazed-binary gratings with $\Lambda_{s}=\lambda_{0} / 2$ and $\lambda_{0} / 5$, respectively.

tion we considered four RCWA diffraction efficiencies obtained for the four largest period-to-wavelength ratios $\left(\Lambda / \lambda_{0}=20,30,40\right.$, and 50$)$. The results of the extrapolation for $N=3$ are given in Table 1. An excellent agreement between the extrapolated efficiencies and those predicted by the approximate model is obtained. The maximum deviation is only $0.4 \%$, a value that remains inferior to the estimated error.

We performed the same computational tasks for TE polarization. The results are reported in Table 2 and in Fig. 4. Once again, excellent agreements between RCWA computational results and the predictions from the approximate model are obtained. Note that the vertical scale in Figs. 3 and 4 has been expanded to visualize relatively small discrepancies between the approximate model and RCWA results.

B. Nonnormal Incidence and Nominal Wavelength In this subsection we study the response of blazed-binary gratings under oblique illumination. We first consider the TM polarization case and two $50 \lambda_{0}$-period blazedbinary gratings. Figure 5 shows the first-transmittedorder diffraction efficiency (computed with RCWA) as a function of the angle of incidence in glass. Pluses correspond to $\Lambda_{s}=\lambda_{0} / 2$, and crosses to $\Lambda_{s}=\lambda_{0} / 5$. The curves represent the asymptotic values $\eta_{1}$ of the efficiency for infinitely large periods. The solid and dashed curves are obtained for $\Lambda_{s}=\lambda_{0} / 2$ and $\lambda_{0} / 5$, respectively. A good agreement between RCWA computational results and the predictions from the approximate model is obtained. The discrepancy is smaller, and usually much smaller, than 4\%. A similar agreement is obtained for the TE polarization case, as shown in Fig. 6.

In Fig. 5 we note that the blazed-binary grating with a sampling period of $\lambda_{0} / 2$ is much more sensitive to the ob- 
lique illumination than the blazed-binary grating with a smaller sampling period $\Lambda_{s}=\lambda_{0} / 5$. There are two reasons for that. First, for $15^{\circ}<\theta_{1}<25^{\circ}$, guided-mode resonances are excited for the $\Lambda_{s}=\lambda_{0} / 2$ case and for fill factors larger than 0.4. These guided-mode resonances result in a drastic decrease of the grating transmission. Of course, they do not exist in the $\Lambda_{s}=\lambda_{0} / 5$ case. Second, the subwavelength gratings seen locally by the incident plane wave for $\Lambda_{s}=\lambda_{0} / 2$ do not behave as zeroorder gratings at any angle of incidence. More specifically, for $\left|\theta_{1}\right|>18^{\circ}$, the reflected negative first diffracted order of these subwavelength gratings becomes nonevanescent in glass. This order is significantly excited, especially for $\left|\theta_{1}\right|>25^{\circ}$. For $\Lambda_{s}=\lambda_{0} / 5$, the non-

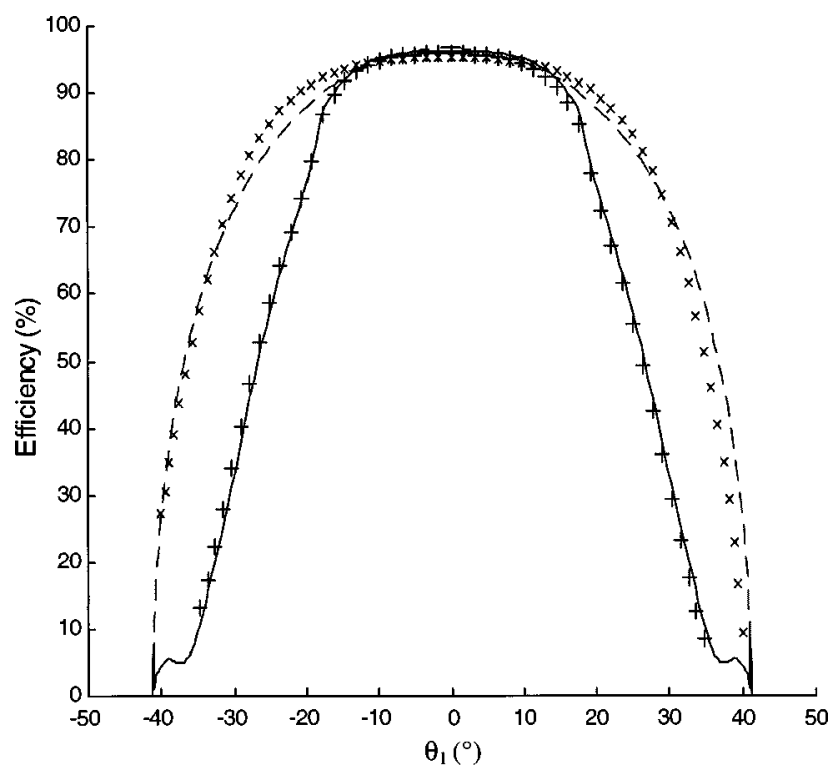

Fig. 6. Same as Fig. 5 but for TE polarization.

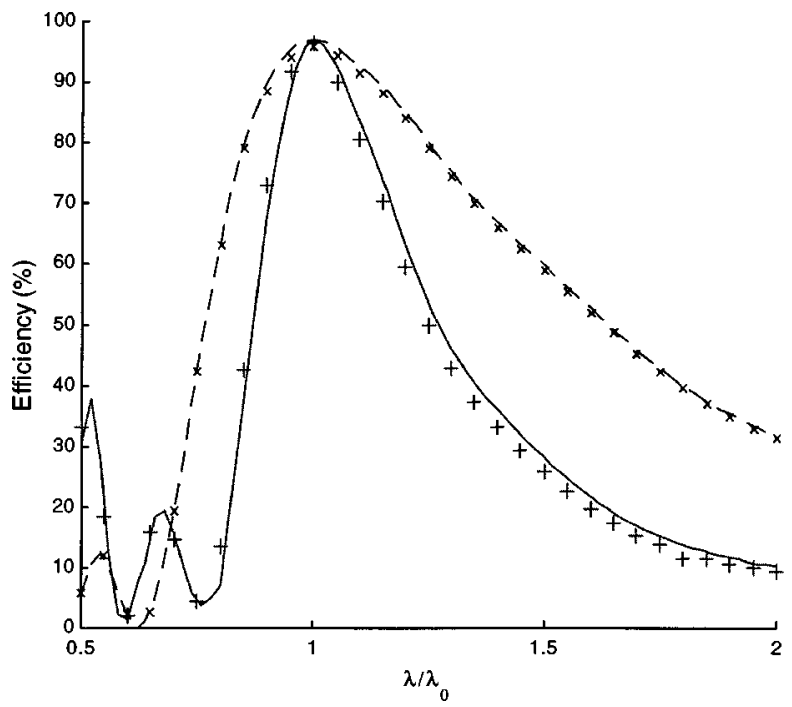

Fig. 7. First-transmitted-order diffraction efficiency versus normalized wavelength $\lambda / \lambda_{0}$ for TM polarization. Solid $\left(\Lambda_{s}\right.$ $\left.=\lambda_{0} / 2\right)$ and dashed $\left(\Lambda_{s}=\lambda_{0} / 5\right)$ curves, asymptotic predictions of the model for $\Lambda / \lambda_{0} \rightarrow \infty$. Pluses and crosses, RCWA computational results obtained for $50 \lambda_{0}$-period blazed-binary gratings with $\Lambda_{s}=\lambda_{0} / 2$ and $\lambda_{0} / 5$, respectively.

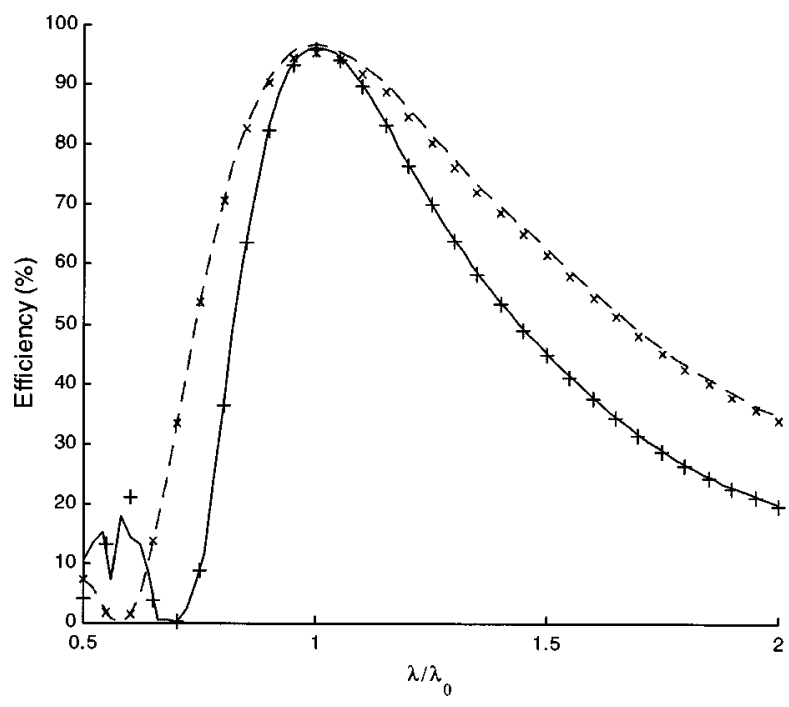

Fig. 8. Same as Fig. 7 but for TE polarization.

zero diffracted orders of the subwavelength gratings are all evanescent. A similar interpretation holds for the TE polarization case in Fig. 6, except that no waveguide resonance is observed in this case.

\section{Normal Incidence and Nonnominal Wavelength}

In this subsection we test the wavelength-dependence of blazed-binary gratings as a function of the normalized wavelength $\left(\lambda / \lambda_{0}\right)$ for TM polarization. We have computed with electromagnetic theory the efficiency of the first-transmitted order of diffraction for a period equal to $50 \lambda_{0}$, represented by the crosses and the pluses in Fig. 7 for $\Lambda_{s}=\lambda_{0} / 2$ and $\lambda_{0} / 5$, respectively. The asymptotic values of the efficiency for infinitely large periods have also been predicted with the approximate model. The results are represented by the curves. The solid and dashed curves hold for $\Lambda_{s}=\lambda_{0} / 2$ and $\lambda_{0} / 5$, respectively. The same computational tasks for TE polarization have been done. The results are reported in Fig. 8. It is clear from these graphs that there is a good agreement of the asymptotic values and the electromagnetic computation for period of 50 wavelengths.

In Fig. 7 we note that the blazed-binary grating with a sampling period of $\lambda_{0} / 2$ is much more sensitive to the wavelength than the blazed-binary grating with a smaller sampling period $\Lambda_{s}=\lambda_{0} / 5$. This higher sensitivity is explained by the fact that the effective index of subwavelength gratings is highly wavelength-dependent for large sampling periods. In general, the effective index $n_{\text {eff }}$ of subwavelength lamellar gratings can be expanded in a power series of $\Lambda_{s} / \lambda_{0}, n_{\text {eff }}=n_{\text {eff } 0}+n_{\text {eff } 2}\left(\Lambda_{s} / \lambda_{0}\right)^{2}$ $+\cdots$, where $n_{\text {eff } 0}$ and $n_{\text {eff } 2}$ depend only on the angle of incidence and on the permittivity contrast; see, for instance, Ref. 9. A similar interpretation holds for the TE polarization case in Fig. 8.

\section{CONCLUSION}

In this work we proposed an approximate model to predict the behavior of one-dimensional blazed-binary structures with local periods much larger than the wavelength. The model is based on both scalar and electromagnetic theory. 
It is rather simple and requires only electromagnetic computations performed on zero-order gratings. The accuracy of the model was tested against RCWA computational results obtained for blazed-binary gratings with large periods. First, the model has been tested for a normal incidence and for nominal design wavelength against asymptotic values extrapolated from RCWA numerical results obtained for blazed-binary gratings with periods increasing up to 50 wavelengths. An excellent agreement between these extrapolated values and the model predictions was obtained. Second, it was shown that the model is able to predict quantitatively the wavelength and incidence-angle dependences of the diffraction efficiency of blazed-binary structures.

This simple model appears to be an efficient tool to predict and optimize the performance of blazed-binary structures dedicated to geometrical aberration correction or achromatization. The model can be applied to many other different diffractive structures, like standard blazed-échelette diffractive elements with coatings, for instance.

\section{ACKNOWLEDGMENTS}

The authors thank M.-P. Jurek and J. Rollin from Thomson Optronique in Guyancourt, France, for motivating this study and for fruitful discussions. Although not reported here, the approximate model has also been validated for blazed-échelette gratings with large periods. The rigorous computation for these gratings was performed by G. Granet from the Laboratoire des Sciences, des Matériaux pour l'Electronique et d'Automatique at Aubière; the authors thank him for his help. Laure Lee is pleased to acknowledge a Ph.D. fellowship from the Délégation Générale pour l'Armement.

\section{REFERENCES}

1. W. Stork, N. Streibl, H. Haidner, and P. Kipfer, "Artificial distributed-index media fabricated by zero-order gratings," Opt. Lett. 16, 1921-1923 (1991).

2. Ph. Lalanne, S. Astilean, P. Chavel, E. Cambril, and H Launois, "Blazed binary subwavelength gratings with efficiencies larger than those of conventional échelette gratings," Opt. Lett. 23, 1081-1083 (1998).

3. Ph. Lalanne, S. Astilean, P. Chavel, E. Cambril, and H. Launois, "Design and fabrication of blazed binary diffractive elements with sampling periods smaller than the structural cutoff," J. Opt. Soc. Am. A 16, 1143-1156 (1999).

4. Ph. Lalanne, "Waveguiding in blazed-binary diffractive elements," J. Opt. Soc. Am. A 16, 2517-2520 (1999).

5. M. Born and E. Wolf, Principles of Optics, 6th ed. (Macmillan, New York, 1964), Chap. 8, p. 387.

6. M. G. Moharam, E. B. Grann, D. A. Pommet, and T. K. Gaylord, "Formulation for stable and efficient implementation of the rigorous coupled-wave analysis of binary gratings," J. Opt. Soc. Am. A 12, 1068-1076 (1995).

7. Ph. Lalanne and G. M. Morris, "Highly improved convergence of the coupled-wave method for TM polarization," J. Opt. Soc. Am. A 13, 779-784 (1996).

8. W. H. Press, B. P. Flannery, S. A. Teukolsky, and W. T. Vetterling, Numerical Recipes: The Art of Scientific Computing (Cambridge U. Press, Cambridge, UK, 1989), Chap. 3.

9. S. M. Rytov, "Electromagnetic properties of a finely stratified medium," Sov. Phys. JETP 2, 466-475 (1956). 